\title{
Exosomes from Suxiao Jiuxin pill-treated cardiac mesenchymal stem cells decrease H3K27 demethylase UTX expression in mouse cardiomyocytes in vitro
}

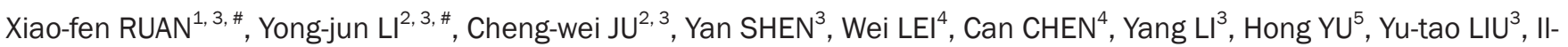 \\ man $\mathrm{KIM}^{3}$, Xiao-long WANG ${ }^{1, *}$, Neal L WEINTRAUB ${ }^{3}$, Yaoliang TANG ${ }^{3, *}$ \\ ${ }^{1}$ Cardiovascular Department, Cardiovascular Research Institute of Traditional Chinese Medicine, Shuguang Hospital of Shanghai \\ University of Traditional Chinese Medicine, Shanghai 201203, China; ${ }^{2}$ Department of Cardiology, Zhongda Hospital, Medical School \\ of Southeast University, Nanjing 210009, China; ${ }^{3}$ Medical College of Georgia, Augusta University, Augusta, GA, USA; ${ }^{4}$ Laboratory of \\ Cardiovascular Diseases, Guangdong Medical University, Zhanjiang 524023, China; ${ }^{5}$ Department of Cardiology, Second Affiliated \\ Hospital, College of Medicine, Zhejiang University, Hangzhou 310009, China
}

\begin{abstract}
Suxiao Jiuxin pill (SJP) is a traditional Chinese medicine for the treatment of acute coronary syndrome in China, which contains two principal components, tetramethylpyrazine (TMP) and borneol (BOR). Thus far, however, the molecular mechanisms underlying the beneficial effects of SJP on the cardiac microenvironment are unknown. Cardiac mesenchymal stem cells (C-MSCs) communicate with cardiomyocytes (CMs) through the release of microvesicles (exosomes) to restore cardiac homeostasis and elicit repair, in part through epigenetic regulatory mechanisms. In this study, we examined whether SJP treatment altered C-MSC-derived exosomes (SJPExos) to cause epigenetic chromatic remodeling in recipient CMs. C-MSC isolated from mouse hearts were pretreated with SJP (SJPExos), TMP (TMP-Exos) or BOR (BOR-Exos). Then, HL-1 cells, a mouse cardiomyocyte line, were treated with exosomes from control C-MSCs (Ctrl-Exos), SJP-Exos, TMP-Exos or BOR-Exos. Treatment with SJP-Exos significantly increased the protein levels of histone 3 lysine 27 trimethylation (H3K27me3), a key epigenetic chromatin marker for cardiac transcriptional suppression, in the HL-1 cells. To further explore the mechanisms of SJP-Exo-mediated H3K27me3 upregulation, we assessed the mRNA expression levels of key histone methylases (EZH1, EZH2 and EED) and demethylases (JMJD3 and UTX) in the exosome-treated HL-1 cells. Treatment with SJP-Exo selectively suppressed UTX expression in the recipient HL-1 cells. Furthermore, PCNA, an endogenous marker of cell replication, was significantly higher in SJP-Exo-treated HL-1 cells than in Ctrl-Exo-treated HL-1 cells. These results show that SJP-Exos increase cardiomyocyte proliferation and demonstrate that SJP can modulate C-MSC-derived exosomes to cause epigenetic chromatin remodeling in recipient cardiomyocytes; consequently, SJP-Exos might be used to promote cardiomyocyte proliferation.
\end{abstract}

Keywords: traditional Chinese medicine; Suxiao Jiuxin pill; tetramethylpyrazine; borneol; cardiac mesenchymal stem cells; exosomes; epigenetic regulation; H3K27 methylases; H3K27 demethylases; UTX

Acta Pharmacologica Sinica (2018) 39: 579-586; doi: 10.1038/aps.2018.18; published online 15 Mar 2018

\section{Introduction}

Suxiao Jiuxin pill (SJP) is a famous traditional Chinese medicine used for treating acute ischemic heart disease. The two major components of SJP are tetramethylpyrazine (TMP) and borneol (BOR) $)^{[1-3]}$. SJP has significant effects on oxidative stress and vascular reactivity that may lead to improved coronary blood flow and chest pain relief. However, the molecular mechanisms through which SJP regulates the cardiac microen-

\footnotetext{
\# These authors contributed equally to this work.

* To whom correspondence should be addressed.

E-mail yaotang@augusta.edu (Yaoliang TANG); wxlqy0214@163.com (Xiao-long WANG)

Received 2017-08-23 Accepted 2018-01-11
}

vironment are unclear.

Hearts are terminally differentiated and have limited proliferative capacity. Therefore, dead cardiomyocytes are primarily replaced with scar tissue after myocardial infarction; this process leads to left ventricular remodeling and heart failure in many patients, despite guideline-directed medical therapy. Invasive therapies, such as coronary artery bypass surgery or percutaneous revascularization, improve blood supply to the ischemic myocardium but cannot regenerate cardiomyocytes. Other options for patients with end stage heart failure include heart transplantation and ventricular assist devices, but these options are limited by their cost and the limited supply of donor hearts. A recent study by Wang et al ${ }^{[4]}$ demonstrated 
that mature adult cardiomyocytes (ACMs) can reenter the cell cycle and form new cardiomyocytes through a dedifferentiation, proliferation and re-differentiation (DPR) process in the infarct border zone. This novel concept provides a promising approach for repairing injured hearts.

Stem cell therapy has consistently led to improvements in cardiac function in animal models of myocardial infarction and in humans with ischemic cardiomyopathy. The beneficial effects of stem cells have primarily been linked to the paracrine mediators they produce, which in turn act on neighboring cardiomyocytes and vascular cells to restore the microenvironment and promote functional recovery ${ }^{[5]}$. Cardiac mesenchymal stem cells (C-MSCs) are heart-derived mesenchymal stromal cells that express the cardiac specific transcription factor GATA- $4^{[6]}$ and have the potential to differentiate into vascular smooth muscle cells ${ }^{[7]}$ and endothelial cells ${ }^{[6,8]}$. C-MSCs play a key role in maintaining cardiac homeostasis and promoting heart repair via paracrine effects, such as inducing angiogenic cytokines ${ }^{[9,10]}$. C-MSC-mediated heart repair is in part mediated by exosomes, $40-100 \mathrm{~nm}$ microvesicles that play a critical role in cell-cell signaling communications ${ }^{[11-13]}$. Exosomes are essential for intercellular communication and are a key mechanism of stem cell-mediated heart repair ${ }^{[11-13]}$. Exosomes contain numerous biologically active components, including proteins, lipids, and microRNA; the latter of which can epigenetically regulate gene expression via cell-cell interactions. However, the role of exosomes in regulating epigenetic chromatin remodeling and how this process might be influenced by commonly prescribed medical therapies are largely unknown; in particular, the potential impact of SJP treatment on C-MSC-mediated cardiomyocyte regulation via exosomes is unclear.

Histone-dependent chromatin remodeling via the trimethylation of histone $\mathrm{H} 3$ on Lys27 (H3K27me3) is essential in controlling stem cell and cardiomyocyte fates ${ }^{[14]}$, including regeneration ${ }^{[15]}$, cardiac reprogramming ${ }^{[16]}$, cell survival and proliferation ${ }^{[17]}$. EZH1 and EZH2, two core catalytic subunits of mammalian Polycomb repressive complex (PRC2), catalyze the addition of a methyl group to histone $\mathrm{H} 3$ at lysine 27, leading to epigenetic repression ${ }^{[18]}$. The dynamic H3K27me3 levels in cells are also controlled by UTX and JMJD3 ${ }^{[19]}$, which regulate muscle differentiation by removing H3K27me3 from the promoter of key transcription factors ${ }^{[20]}$.

In this study, we compared H3K27me3 levels in cardiomyocytes after C-MSC-derived exosome treatment. Compared with control C-MSC-derived exosomes (Ctrl-Exos), SJP preconditioned exosomes (SJP-Exos) significantly increased H3K27me3 levels in recipient cardiomyocytes and downregulated UTX gene expression, indicating that SJP-Exos regulate H3K27 demethylation in cardiomyocytes. Importantly, SJPExo treatment increased PCNA (an endogenous cell proliferation marker) expression in recipient cardiomyocytes.

\section{Materials and methods}

\section{C-MSC isolation and cell culture}

C-MSC were isolated from the hearts of 2 to 3 -month-old male C57BL/ 6 mice (The Jackson Laboratory, Bar Harbor, ME, USA) via a 2-step procedure as described previously ${ }^{[6,8]}$. Briefly, in step 1, ventricular cardiac tissue was minced into $1 \mathrm{~mm}^{3}$ pieces, digested with $0.1 \%$ collagenase IV and $1 \mathrm{U} /$ $\mathrm{mL}$ dispase in DMEM/F-12, and explanted into fibronectin/ gelatin-coated wells $(0.5 \mathrm{mg}$ of fibronectin in $100 \mathrm{~mL}$ of $0.1 \%$ gelatin). We maintained the cardiac explant cultures until the small, round, phase-bright cells migrated from the adherent explants and proliferated over the fibroblast layer. In step 2, Sca- $1+$ cells were purified from the phase-bright cells by using a mouse hematopoietic lineage-depletion cocktail kit (Stemcell Technologies, Vancouver, Canada), followed by magnetic-activated cell sorting (MACS) using Sca-1 magnetic beads (Miltenyi Biotec Inc, Auburn, CA, USA) as instructed by the manufacturers' protocols. The sorted Sca-1 cells were cultured and maintained in complete media (DMEM/F12, $10 \%$ fetal bovine serum (FBS), $200 \mathrm{mmol} / \mathrm{L}$ L-glutamine, $55 \mathrm{nmol} / \mathrm{L} \beta$-mercaptoethanol, and 1\% MEM non-essential amino acids).

\section{Drug preparation, C-MSC preconditioning and exosome purification}

The origin, medicinal composites, and processing technology of SJP (Tianjin Zhongxin Pharmaceutical Group Co, Ltd, Tianjin, China) were strictly standardized based on marker compounds to achieve quality control according to the Chinese Pharmacopoeia $2015^{[21]}$. The dose selections for SJP and BOR in cell culture were based upon therapeutically effective plasma drug concentrations in patients ${ }^{[22,23]}$; the dosage of TMP for cell culture was based on a previous experimental report ${ }^{[24]}$. SJP, TMP and BOR were diluted in ethanol to $62.5 \mathrm{mg} / \mathrm{mL}$, $25 \mathrm{mg} / \mathrm{mL}$ and $15 \mathrm{mg} / \mathrm{mL}$, respectively, as $1000 \times$ stock solutions for the cell culture experiments. For C-MSC preconditioning, C-MSCs were treated with $0.1 \%$ ethanol (Ctrl), 62.5 $\mu \mathrm{g} / \mathrm{mL}$ SJP (SJP), $25 \mu \mathrm{g} / \mathrm{mL}$ TMP or $15 \mu \mathrm{g} / \mathrm{mL}$ BOR in culture medium with $10 \%$ exosome-depleted FBS for $48 \mathrm{~h}$. The C-MSC exosomes were purified as described previously, with minor modifications ${ }^{[12,25,26]}$. Briefly, supernatants were centrifuged at 1000 rounds per minute for $10 \mathrm{~min}$ to separate out the cells; next, the supernatants were filtered through a $0.22 \mu \mathrm{m}$ filter to remove any cell debris. Exosomes in medium were precipitated with 5× polyethylene glycol 4000 (PEG4000, 8.5\% final concentration) with $10 \times \mathrm{NaCl}(0.4 \mathrm{~mol} / \mathrm{L}$ final concentration) overnight at $4{ }^{\circ} \mathrm{C}$; then, the samples were centrifuged at $2100 \times \mathrm{g}$ for $30 \mathrm{~min}$, and the pellets were resuspended in PBS and stored at $-80^{\circ} \mathrm{C}$ until usage.

\section{Electron microscopy and Zeta analysis}

For the transmission electron microscopy (TEM) morphology assessments, $3 \mu \mathrm{L}$ of the exosome pellet was placed on formvar carbon-coated 200 mesh copper electron microscopy grids, incubated for $5 \mathrm{~min}$ at room temperature (RT), and then subjected to standard uranyl acetate staining ${ }^{[27]}$. The grid was washed with three aliquots of PBS and allowed to become semi-dry at room temperature before observation by transmission electron microscope (JEOL JEM 1230, Peabody, MA, USA). Micrographs were used to quantify the diameters 
of the exosomes. We measured the exosome particle sizes by nanoparticle tracking analysis (NTA) with ZetaView PMX 110 (Particle Metrix, Meerbusch, Germany) and the corresponding software ZetaView 8.02.28. The ZetaView system was calibrated using $100 \mathrm{~nm}$ polystyrene particles.

\section{Exosomal transfer to $\mathrm{HL}-1$ cells}

The murine cardiomyocyte cell line HL-1 (a kind gift from Prof Claycomb) was cultured in gelatin/fibronectin-coated 6 well plates with Claycomb Medium (Sigma-Aldrich) supplemented with $10 \%$ exosome-depleted FBS, $100 \mathrm{U} / \mathrm{mL}$ penicillin, $100 \mu \mathrm{g} / \mathrm{mL}$ streptomycin, $0.1 \mathrm{mmol} / \mathrm{L}$ norepinephrine (SigmaAldrich) and $2 \mathrm{mmol} / \mathrm{L}$ L-glutamine (Sigma-Aldrich). HL-1 cells in each 6 well plate were treated with $250 \mu \mathrm{g}$ of Ctrl-Exos, SJP-Exos, TMP-Exos or BOR-Exos for $24 \mathrm{~h}$; RNA and protein were extracted after exosome treatment. The exosome doses used in this study are in accordance with the recommendations of System Biosciences (SBI), which suggest the use of 250 $\mu \mathrm{g}$ of exosomes to treat cells in a 6 well plate format. To determine the effects of $\mathrm{H}_{2} \mathrm{O}_{2}$ on apoptosis, HL- 1 cells were treated with $1 \mathrm{mmol} / \mathrm{L} \mathrm{H}_{2} \mathrm{O}_{2}$ in DMEM for $2 \mathrm{~h}$.

\section{Isolation and quantification of mRNA}

Total RNA from HL-1 cells was extracted by RNAzol RT (Molecular Research Center, Inc, Cincinnati, OH, USA) following the manufacturer's instructions. cDNA was synthesized from total RNA by using the RevertAid First Strand cDNA Synthesis kit (Thermo Scientific). The synthesized cDNA was used to perform quantitative PCR on a CFX96 Touch Real-Time PCR Detection System (Bio-Rad) using PowerUp SYBR ${ }^{\circledR}$ Green Master Mix (ThermoFisher). Amplification was performed at $50{ }^{\circ} \mathrm{C}$ for $2 \mathrm{~min}, 95^{\circ} \mathrm{C}$ for $2 \mathrm{~min}$, 40 cycles of $95^{\circ} \mathrm{C}$ for $15 \mathrm{~s}$, and $60^{\circ} \mathrm{C}$ for 1 min with the indicated primers (Table 1).

\section{Western blotting assay}

Purified exosomes or exosome-treated HL-1 cells were assessed for protein content using a BCA protein assay kit

Table 1. Prime list.

\begin{tabular}{ll}
\hline Gene list & \multicolumn{1}{c}{ Sequence $\left(5^{\prime}-3^{\prime}\right)$} \\
\hline EZH1 FWD & AGCTTCCTCTTCAACCTCAAC \\
EZH1 REV & CACCATAACCACTTTGGCATAAC \\
EZH2 FWD & GGTTAATGGTGACCACAGGATAG \\
EZH2 REV & CGTTCGATGCCCACATACTT \\
EED FWD & CTGTGGGAAGCAACAGAGTAA \\
EED REV & TAGGTCCATGCACAAGTGAAA \\
JMJD3 FWD & CACCCAAGAAGAGGAGAAGAAG \\
JMJD3 REV & AGAACAGAGGCCAACGATTT \\
UTX FWD & CAGCAACACCTTCTCCTAAGTC \\
UTX REV & GGGCTCTGAGATTCTTCCATTC \\
GAPDH FWD & TGACATCAAGAAGGTGGTGAAG \\
GAPDH REV & AGTGGGAGTTGCTGTTGAAG \\
\hline
\end{tabular}

(Pierce, Rockford, IL, USA). Proteins were resolved on $10 \%$ sodium dodecyl sulfate bis-tris gels and transferred onto Odyssey ${ }^{\circledR}$ nitrocellulose membranes (LI-COR Biosicences). The membranes were blocked with Odyssey blocking buffer (LICOR Biosciences, Lincoln, NE, USA) and probed with rabbit anti-CD63 (1:250, Santa Cruz Biotechnology, Inc, Santa Cruz, CA, USA), rabbit anti-CD81 (1:1000, Thermo Scientific), mouse anti-Tsg101 (1:1000, Thermo Scientific), rabbit anti-H3K27me3 (1:1000, Cell Signaling Technology), rabbit anti-UTX (1:1000, Cell Signaling Technology), rabbit anti-cleaved PARP (1:1000, Cell Signaling Technology), rabbit anti-PCNA (1:1000, Cell Signaling Technology), and mouse anti-GAPDH (1:5000, Millipore) at $4{ }^{\circ} \mathrm{C}$ overnight; then, the membranes were incubated for $1 \mathrm{~h}$ at room temperature with IRDye 680 goat anti-rabbit IgG at 1:10 000 or IRDye 800 goat anti-mouse IgG at 1:10 000 (LICOR Biosciences). The probed blots were scanned using an Odyssey infrared imager.

\section{Statistical analysis}

All values are expressed as the mean \pm standard error of the mean (SEM). Student's $t$-test was used to compare two groups. One-way ANOVA was used for comparisons between 3 or more means. A value of $P<0.05$ was considered to indicate a statistically significant difference.

\section{Results}

\section{Characterization of C-MSCs}

C-MSCs were isolated by using a two-step procedure that we described previously with modifications ${ }^{[28-30]}$ : enzymatically digested, minced adult mouse hearts were cultured in fibronectin/gelatin coated plates. After two weeks, cardiacderived cells were collected by light enzymatic digestion, and C-MSCs were enriched by depleting hematopoietic cells using a hematopoietic lineage-depletion cocktail; subsequently, the samples were enriched in Sca-1+ cells via MACS sorting (Figure 1A). Immunofluorescent staining demonstrated that the C-MSCs expressed GATA4, an early cardiac transcription factor, confirming their cardiacspecific origin (Figure 1B). Flow cytometry assays showed that C-MSCs expressed high levels of the MSC-specific cell surface markers Sca-1 and CD105 and very low levels of the hematopoietic maker CD45 (Figure 1C). Taken together, these data indicate that C-MSCs represent a subpopulation of cardiac-derived mesenchymal stem cells.

\section{Characterization of the C-MSC-derived exosomes}

Morphological analysis of the C-MSC-derived pellets using transmission electron microscopy confirmed the presence of exosomes (Figure 2A). Western blot analysis of C-MSCderived exosomes revealed the presence of the typical exosome markers CD63, CD81 and Tsg101 (Figure 2B). The sizes of the isolated exosomes/microvesicles were measured with dynamic light scattering (DLS) using ZetaView ${ }^{\circledR}$, a nanoparticle tracking analyzer of hydrodynamic particle size. As shown in Figure 2C, the peak size of the purified particles was approximately $100 \mathrm{~nm}$. 

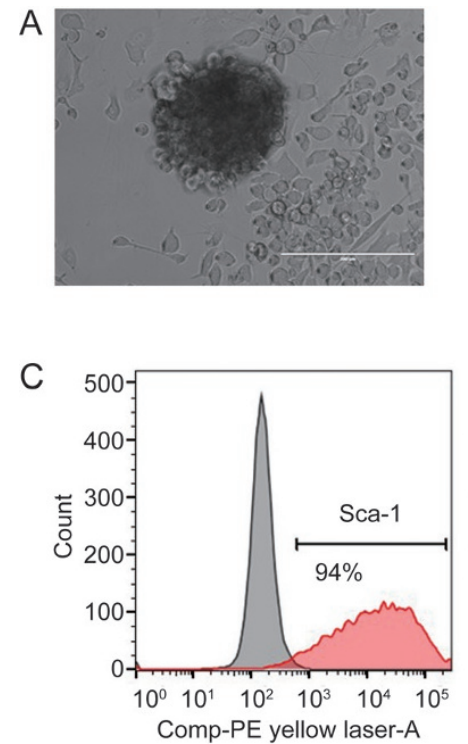

B

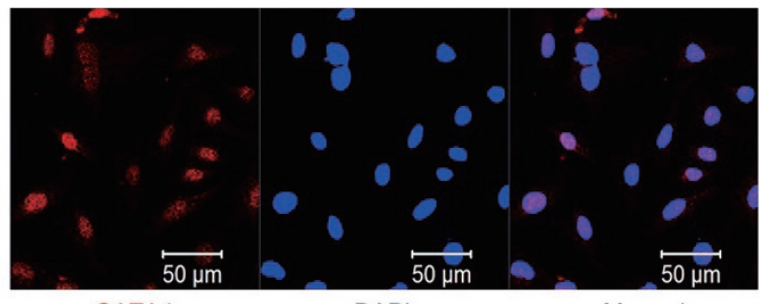

GATA4

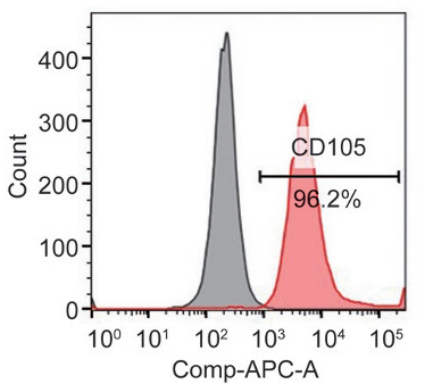

Figure 1. Phenotypic characterization of C-MSCs. (A) Cultured C-MSCs. (B) Immunofluorescent staining of C-MSCs for the expression of the cardiac transcription factor GATA4 (red); cell nuclei were counterstained with DAPI (blue). (C) Flow cytometric analyses of C-MSCs for the expression of the cell surface markers Sca-1, CD105, and CD45.

Effects of C-MSC-derived exosomes on H3K27 trimethylation in cardiomyocytes

To explore the effect of SJP preconditioning on the capacity of C-MSC-derived exosomes to regulate epigenetic chromatin remodeling in cardiomyocytes, $250 \mu \mathrm{g}$ of exosomes from the control cells (Ctrl-Exos) or SJP-treated cells (SJP-Exos) was applied to HL-1 cells in 6 well plates. SJP-Exo application, compared with Ctrl-Exo application, resulted in increased H3K27me3 levels in the HL-1 cells. To understand whether TMP and/or BOR, two major components of SJP, have effects similar to SJP preconditioning in HL-1 cells, we compared H3K27me3 levels in HL-1 cells following the application of exosomes collected from SJP-, TMP- and BOR-treated C-MSCs; we found that both TMP-Exos and BOR-Exos produced effects similar to those of SJP-Exos on H3K27me3 levels in HL-1 cells
(Figure 3). Interestingly, compared with control treatment, SJP, TMP, and BOR treatment did not directly increase H3K27me3 levels in HL-1 cells (Supplemental Figure S1A, S1B).

Identification of the underlying mechanism of SJP-Exo-mediated H3K27 trimethylation in cardiomyocytes

Both histone methylases and histone demethylases can control H3K27me3 levels in cardiomyocytes. To identify how SJPExo application increased H3K27me3 levels in HL-1 cells, we measured the expression of $\mathrm{H} 3 \mathrm{~K} 27$ methylation-related genes (EZH1, EZH2 and EED) and H3K27 demethylation-related genes (JMJD3 and UTX). Using qRT-PCR assays, we found that SJP-Exo application had minimal effects on the expression of all three H3K27 methylation-related genes (Figure 4A-4C) and the H3K27 demethylase JMJD3 (Figure 4D) in HL-1
A

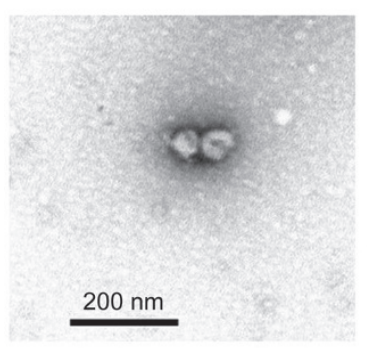

B

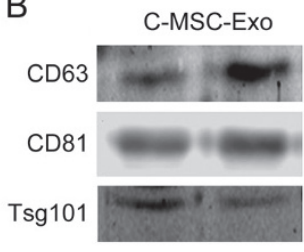

C

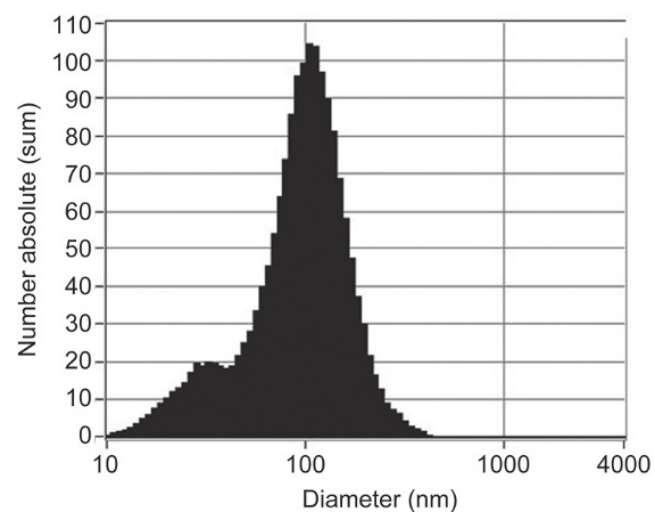

Figure 2. Characterization of C-MSC-derived exosomes. (A) Transmission electron microscopic morphological analysis of exosomes isolated from C-MSCs. Scale bar=200 nm. (B) Western blot results demonstrate the expression of CD63, CD81 and Tsg101 in exosomes derived from C-MSCs. (C) Particle size distribution in purified pellets measured by ZetaView ${ }^{\circledR}$ Particle Tracking Analyzer. 
A

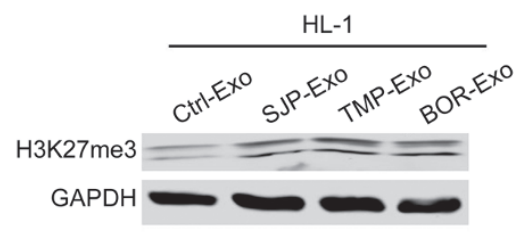

B

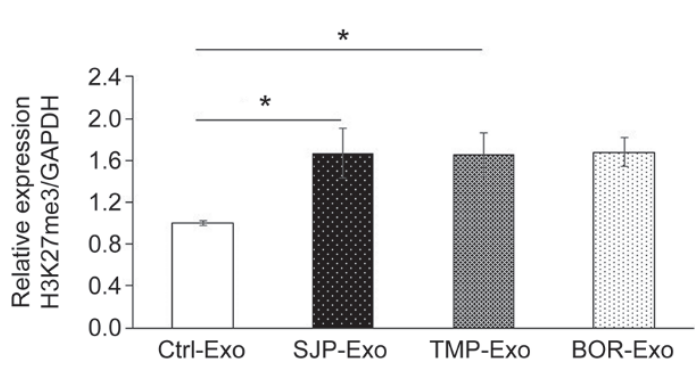

Figure 3. Trimethylation of H3K27 in $\mathrm{HL}-1$ cells following the application of C-MSC-derived exosomes. Western blot analyses show a global increase in H3K27 trimethylation in HL-1 cells after the application of SJP-Exos, TMP-Exos or BOR-Exos in comparison with Ctrl-Exos ( $\left.{ }^{*} P<0.05, n=3\right)$.

cells. However, SJP-Exo application significantly reduced the expression of the H3K27 demethylase UTX (by approximately $60 \%$ ) (Figure 4E). In agreement with the qRT-PCR data, Western blots showed that SJP-Exos significantly reduced UTX pro- tein levels in HL-1 cells (Figure 4F, 4G). This finding suggests that the application of SJP-Exos increases H3K27me3 in HL-1 cells by inhibiting the histone demethylase UTX. Moreover, we compared the effects of SJP-Exos with those of TMP-Exos
A

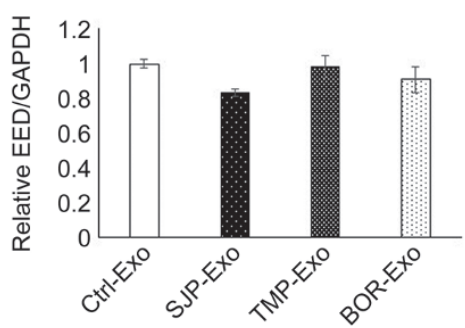

D

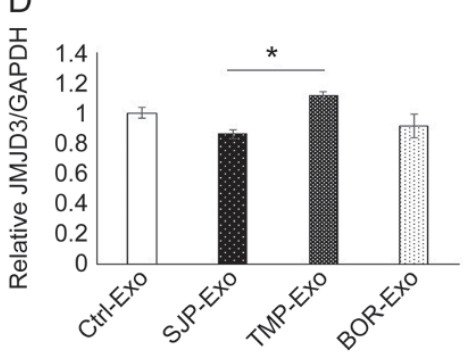

$\mathrm{F}$

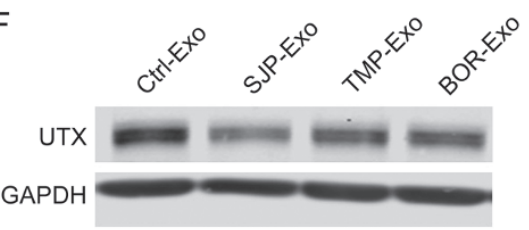

B

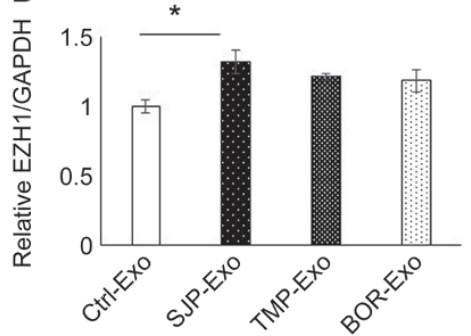

E

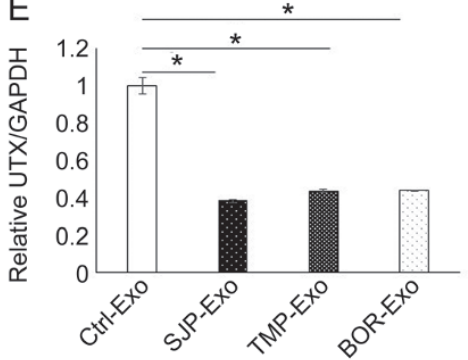

G

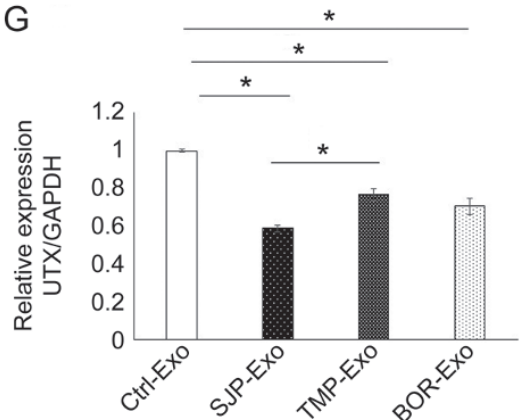

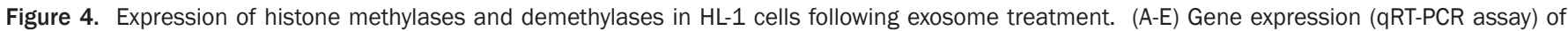

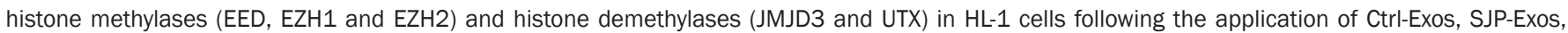

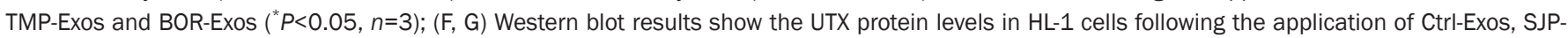

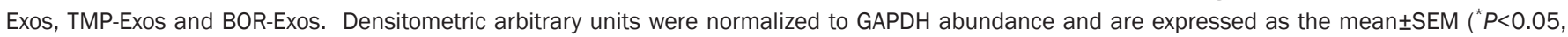
$n=3)$. 
and BOR-Exos on UTX expression in HL-1 cells. We found that compared with TMP-Exo treatment, SJP-Exos significantly reduced the UTX levels in cardiomyocytes (Figure 4F, 4G). We further measured the dose-response and time-response of UTX expression levels to SJP-Exo treatment in HL-1 cells. As shown in Supplemental Figure S2A, SJP-Exo-mediated UTX reductions were detected by Western blot at $125 \mu \mathrm{g}$, and the $250 \mu \mathrm{g}$ and $500 \mu \mathrm{g}$ doses significantly reduced the UTX levels in HL-1 cells $(P<0.05, n=3)$. Next, we studied the time-response of UTX expression levels to SJP-Exo treatment in HL-1 cells. We observed that in comparison with $12 \mathrm{~h}$ treatment, $24 \mathrm{~h} \mathrm{SJP-}$ Exo treatment significantly reduced the UTX levels in HL-1 cells, but $48 \mathrm{~h}$ treatment did not further decrease these levels (Supplemental Figure S2B).

Identification of the functional consequences of SJP-Exo treatment in cardiomyocytes

To determine the function of SJP-Exos in cardiomyocytes, we first tested whether SJP-Exo treatment affects $\mathrm{H}_{2} \mathrm{O}_{2}$-induced apoptosis in cardiomyocytes. To do this, we compared apoptosis in cardiomyocytes with/without oxidative stress. As shown in Figure 5A, compared with Ctrl-Exo treatment, SJPExo treatment did not reduce the levels of cleaved PARP (a marker for apoptosis) either at baseline or following treatment with $\mathrm{H}_{2} \mathrm{O}_{2}$. We next studied whether SJP-Exo treatment affected cardiomyocyte proliferation; to do this, we measured the levels of PCNA (an endogenous marker for cell proliferation) expression in HL-1 cells. We observed that compared with Ctrl or Ctrl-Exo groups, SJP-Exo treatment significantly increased the PCNA protein levels in HL-1 cells (Figure 5B), indicating that SJP-Exo treatment promoted cardiac proliferation.

\section{Discussion}

Suxiao Jiuxin pill (Supplemental Figure S3) has multiple ben- eficial effects on vascular function and is widely used to treat heart disease. Here, we demonstrate a novel function of SJP through which it can mediate the ability of cardiac mesenchymal stem cell-derived exosomes to regulate the levels of H3K27me3, a key epigenetic chromatin marker for cardiac transcriptional suppression, in HL-1 cardiomyocytes and to promote cardiomyocyte proliferation. The major mechanism appears to be related to the downregulation of the expression of the demethylase UTX. These functions of SJP are shared by its two major herbal components, TMP and BOR. Our findings have implications regarding the mechanisms of the beneficial effects of SJP in treating heart disease patients.

The trimethylation of histone 3 lysine 27 modulates cardiomyocyte proliferation. H3K27me3 levels are controlled by balancing H3K27 methyltransferases (EZH1, EZH2, EED) and demethylases (UTX and JMJD3). The enhancer of zeste homolog 2 (EZH2) is an essential catalytic subunit of Polycomb repressive complex 2 (PRC2), which is responsible for $\mathrm{H} 3 \mathrm{~K} 27 \mathrm{me}^{[31]}$ and the transcriptional repression of targeted genes by inhibiting the binding of acetyltransferases, such as p300 and CBP, to Polycomb group (PcG) target genes ${ }^{[32]}$. Recent studies demonstrate that EZH2-mediated H3K27me3 plays a critical role in heart development, and $\mathrm{Nkx2.5}$-specific EZH2 knockout leads to lethal congenital heart defects via the inhibition of cardiomyocyte proliferation ${ }^{[33]}$. EZH2 overexpression increases $\mathrm{H} 3 \mathrm{~K} 27 \mathrm{me} 3$ levels and promotes the proliferation and migration of stem and progenitor cells ${ }^{[34]}$. UTX is one of two H3K27me3 demethylases that remove the suppressive H3K27me3 marks. In this study, we found that SJP functionally alters the exosomes produced by C-MSCs; when these exosomes are applied to HL-1 cardiomyocytes, they cause significant increases in the protein levels of H3K27me3 (Figure 6). Mechanistic studies demonstrated that the SJP-Exos had little effect on the transcripts of key histone methyltransferases
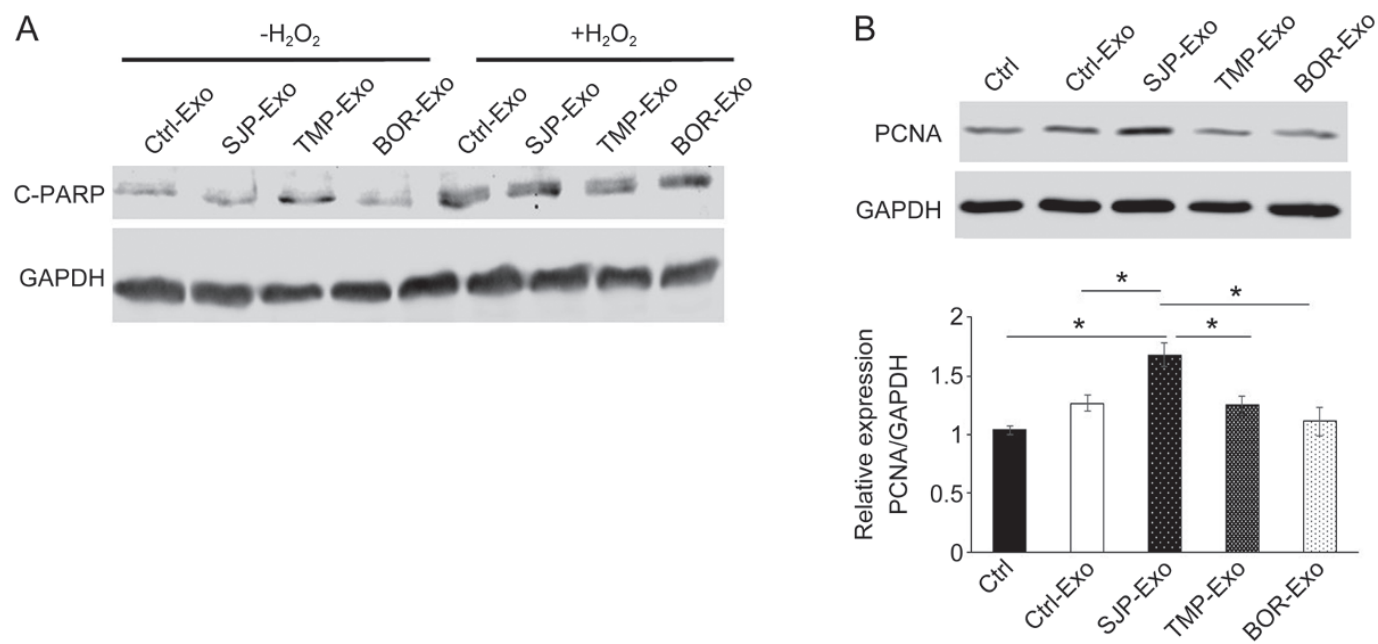

Figure 5. Effect of SJP-Exos on $\mathrm{H}_{2} \mathrm{O}_{2}$-induced apoptosis and proliferation in HL-1 cells. (A) Western blot of cleaved PARP (C-PARP) expression in HL-1 cells following overnight treatment with Ctrl-Exos, SJP-Exos, TMP-Exos and BOR-Exos and with/without 1 mmol/L $\mathrm{H}_{2} \mathrm{O}_{2}$ treatment for 2 h; (B) Western blot of the expression of the proliferation marker PCNA in HL-1 cells following the application of Ctrl, Ctrl-Exos, SJP-Exos, TMP-Exos and BOR-Exos ( ${ }^{*} P<0.05$, $n=3$ ). 


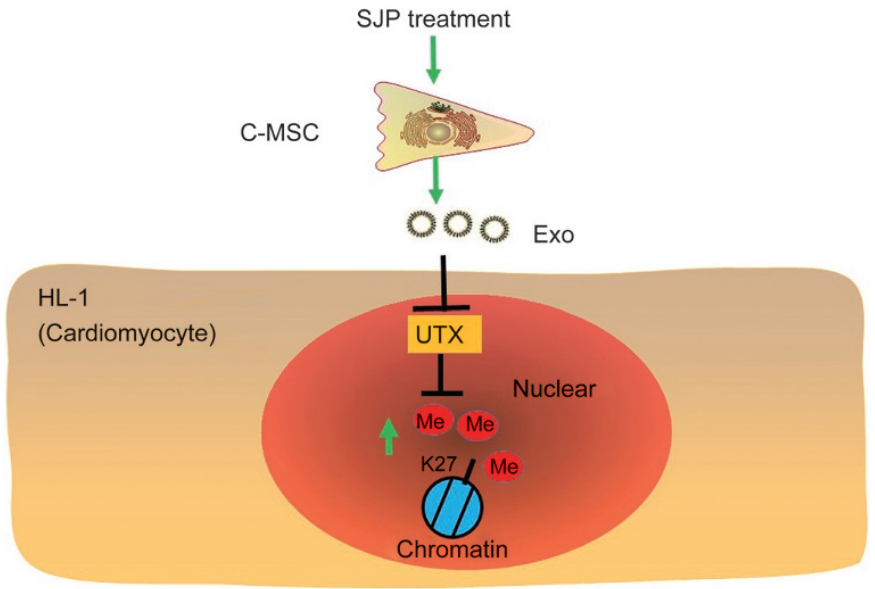

Figure 6. Schematic overview of SJP preconditioned C-MSC-derived exosomes in regulating $\mathrm{H} 3 \mathrm{~K} 27 \mathrm{me} 3$ levels in recipient cardiomyocytes. Mechanistically, SJP-Exo application leads to decreased expression of the histone demethylase UTX in the recipient cardiomyocytes.

(EED, EZH1 and EZH2) or the histone demethylase JMJD3. In contrast, expression of the histone demethylase UTX was dramatically reduced by the SJP-Exos, which may explain why the application of these exosomes increased H3K27me3 levels in HL-1 cardiomyocytes. The specific mechanism for these exosomes in regulating the expression of UTX is unclear. Since exosomes contain numerous non-coding RNAs, including miRNAs, lncRNAs and circRNAs, that might modulate UTX expression via the gene network, the final effect may result from both the direct and indirect effects of cross talk among multiple signaling and noncoding RNAs ${ }^{[35]}$; therefore, integrated analyses of these noncoding RNAs are needed to understand their regulatory machinery.

In this study, we observed that both TMP-Exos and BOR-Exos produced effects similar to those of SJP-Exo on H3K27me3 levels in HL-1 cells; these results suggest that the major components of SJP are equally effective at regulating the function of C-MSC-derived exosomes to elicit epigenetic chromatin modifications in recipient cardiomyocytes. We also observed that compared with TMP-Exo treatment, SJP-Exos significantly reduced the UTX levels in cardiomyocytes, suggesting that the multi-component herbal compound might be more effective at regulating exosome-mediated epigenetic chromatin remodeling than each individual herb component. The concept of "synergy" proposed by Traditional Chinese Medicine refers to the synergistic effects of multiple-component herbal combinations that lead to enhanced pharmacological responses. In traditional Chinese medicine practice, multiherb medicines are prescribed to patients with cardiovascular diseases based on their synergistic, multi-targeted interactive effects $^{[36]}$.

As shown by our experiments, SJP-Exo treatment does not protect cardiomyocytes from apoptosis caused by $\mathrm{H}_{2} \mathrm{O}_{2}$ mediated oxidative stress; however, SJP-Exo treatment can increase the levels of PCNA, a proliferation marker expressed in cardiomyocytes, suggesting that SJP-Exos might be used to stimulate cardiac proliferation. In future studies, we might use SJP-Exos to treat heart failure by delivering the exosomes directly to the cardiomyocytes. This strategy for promoting cardiomyocyte proliferation might improve cardiac repair.

In this study, we used a polyethylene glycol (PEG)-based method that we described previously to purify exosomes ${ }^{[12]}$. Compared with the gold-standard differential centrifugation and sucrose gradient purification methods, PEG-based exosome purification has been demonstrated to have the advantages of preserving RNA cargo, not inhibiting biological activity, and enriching highly pure exosomes ${ }^{[37]}$. Our enriched exosomes were confirmed to express the CD63, CD81 and Tsg101 markers by Western blot analyses (Figure 2B); however, PEG-based methods cannot differentiate the exosomes from microvesicles.

In summary, we demonstrated that SJP can regulate C-MSCderived exosome functions to downregulate the expression of the demethylase UTX, thus increasing H3K27me3 levels in recipient cardiomyocytes. Most importantly, SJP-Exos increased PCNA expression in cardiomyocytes. This finding broadens our current understanding of the molecular mechanisms of SJP in treating ischemic heart diseases and highlights for the first time the role of stem cell-derived exosomes in epigenetically remodeling chromatin in recipient cells.

\section{Acknowledgements}

This study was supported by the National Natural Science Foundation of China (No 81403352, 81573647 to Xiao-long WANG and Xiao-fen RUAN, and 81528002 to Yao-liang TANG), the Shanghai Key Laboratory of Traditional Chinese Clinical Medicine (№ 14DZ2273200); this study was also partially supported by the Tianjin Zhongxin Pharmaceutical Group Co, Ltd, Tianjin, China.

Il-man KIM, Neal L WEINTRAUB, and Yao-liang TANG were partially supported by the American Heart Association GRNT31430008, NIH-AR070029, NIH-HL086555, NIHHL134354, and NIH-HL12425.

\section{Author contribution}

Conception and design: Xiao-long WANG, Yaoliang TANG; Data collection: Xiao-fen RUAN, Yong-jun LI, Cheng-wei JU, Yan SHEN; Data analysis: Xiao-fen RUAN, Xiao-long WANG; Data interpretation: Xiao-fen RUAN, Wei LEI, Can CHEN, Xiao-long WANG, Yaoliang TANG; Manuscript writing: Ilman KIM, Hong YU, Neal L WEINTRAUB, Yaoliang TANG.

\section{Supplementary information}

Supplementary information is available at the website of Acta Pharmacologica Sinica.

\section{References}

1 Lu Z, Zhang Y, Zhuang P, Zhang J, Zhou H, Zhang M, et al. Protective effect of Suxiao jiuxin pill, a traditional Chinese medicine, against acute myocardial ischemia in dogs. BMC Complement Altern Med 2015; 15: 373.

2 Ren Y, Li D, Zheng H, Lv J, Leng J, Zhang L, et al. Acupoint application in patients with chronic stable angina pectoris: study protocol of a 
randomized, double-blind, controlled trial. Evid Based Complement Alternat Med 2014; 2014: 619706.

3 Zhang J, Zhuang P, Lu Z, Zhang M, Zhang T, Zhang Y, et al. Suxiaojiuxin pill enhances atherosclerotic plaque stability by modulating the MMPs/TIMPs balance in ApoE-deficient mice. J Cardiovasc Pharmacol 2014; 64: 120-6.

4 Wang WE, Li L, Xia X, Fu W, Liao Q, Lan C, et al. Dedifferentiation, proliferation and redifferentiation of adult mammalian cardiomyocytes after ischemic injury. Circulation 2017; 136: 834-48.

5 Tang YL, Wang YJ, Chen L, Pan YH, Zhang L, Weintraub NL. Cardiacderived stem cell-based therapy for heart failure: progress and clinical applications. Exp Biol Med (Maywood) 2013; 238: 294-300.

6 Tang YL, Shen L, Qian K, Phillips MI. A novel two-step procedure to expand cardiac Sca-1+ cells clonally. Biochem Biophys Res Commun 2007; 359: 877-83.

7 Chen L, Ashraf M, Wang Y, Zhou M, Zhang J, Qin G, et al. The role of notch 1 activation in cardiosphere derived cell differentiation. Stem Cells Dev 2012; 21: 2122-9.

8 Tang YL, Zhu W, Cheng M, Chen L, Zhang J, Sun T, et al. Hypoxic preconditioning enhances the benefit of cardiac progenitor cell therapy for treatment of myocardial infarction by inducing CXCR4 expression. Circ Res 2009; 104: 1209-16.

9 Tang YL, Zhao Q, Qin X, Shen L, Cheng L, Ge J, et al. Paracrine action enhances the effects of autologous mesenchymal stem cell transplantation on vascular regeneration in rat model of myocardial infarction. Ann Thorac Surg 2005; 80: 229-36.

10 Tang YL, Zhao Q, Zhang YC, Cheng L, Liu M, Shi J, et al. Autologous mesenchymal stem cell transplantation induce VEGF and neovascularization in ischemic myocardium. Regul Pept 2004; 117: 3-10.

11 Campbell CR, Berman AE, Weintraub NL, Tang YL. Electrical stimulation to optimize cardioprotective exosomes from cardiac stem cells. Med Hypotheses 2016; 88: 6-9.

12 Wang Y, Zhang L, Li Y, Chen L, Wang X, Guo W, et al. Exosomes/ microvesicles from induced pluripotent stem cells deliver cardioprotective miRNAs and prevent cardiomyocyte apoptosis in the ischemic myocardium. Int J Cardiol 2015; 192: 61-9.

13 Chen L, Wang Y, Pan Y, Zhang L, Shen C, Qin G, et al. Cardiac progenitor-derived exosomes protect ischemic myocardium from acute ischemia/reperfusion injury. Biochem Biophys Res Commun 2013; 431: 566-71.

14 Juan AH, Wang S, Ko KD, Zare H, Tsai PF, Feng X, et al. Roles of H3K27me2 and H3K27me3 examined during fate specification of embryonic stem cells. Cell Rep 2016; 17: 1369-82.

15 Ai S, Yu X, Li Y, Peng Y, Li C, Yue Y, et al. Divergent requirements for EZH1 in heart development versus regeneration. Circ Res 2017; 121: 106-12.

16 Talkhabi M, Zonooz ER, Baharvand $\mathrm{H}$. Boosters and barriers for direct cardiac reprogramming. Life Sci 2017; 178: 70-86.

17 Chen L, Ma Y, Kim EY, Yu W, Schwartz RJ, Qian L, et al. Conditional ablation of Ezh2 in murine hearts reveals its essential roles in endocardial cushion formation, cardiomyocyte proliferation and survival. PLoS One 2012; 7: e31005.

18 Bodega B, Marasca F, Ranzani V, Cherubini A, Della Valle F, Neguembor MV, et al. A cytosolic Ezh1 isoform modulates a PRC2Ezh1 epigenetic adaptive response in postmitotic cells. Nat Struct Mol Biol 2017; 24: 444-52.

19 Dal-Pra S, Hodgkinson CP, Mirotsou M, Kirste I, Dzau VJ. Demethylation of H3K27 is essential for the induction of direct cardiac reprogramming by miR combo. Circ Res 2017; 120: 1403-13.
20 Liu L, Rando TA. UTX in muscle regeneration--the right dose and the right time. J Clin Invest 2016; 126: 1233-5.

21 National Pharmacopoeia Committee. Chinese Pharmacopoeia 2015, 2015.

22 Wang XL, Liu YM, Zhu GJ. Effects of Suxiao jiuxin pill on patients with acute coronary syndrome undergoing early percutaneous coronary intervention. Zhongguo Zhong Xi Yi Jie He Za Zhi 2012; 32: 1483-7.

23 Guo J, Meng H, Wang L, Zhang L, Hang X, Xu R. Effect of blood drug level change of Borneol in Suxiaojiuxin pill on patients with myocardial ischemia. Modern J Integr Tradit Chin Western Med 2004; 13: 2387 402.

24 Yu L, Li M, She T, Han L. Effects of Tetramethylpyrazine on Ang IIinduced cardiomyocyte hypertrophy and the underlying mechanisms. Chin J Exp Tradit Med Form 2013; 19: 154-7.

25 Lee C, Mitsialis SA, Aslam M, Vitali SH, Vergadi E, Konstantinou G, et al. Exosomes mediate the cytoprotective action of mesenchymal stromal cells on hypoxia-induced pulmonary hypertension. Circulation 2012; 126: 2601-11.

26 Chen Z, Li Y, Yu H, Shen Y, Ju C, Ma G, et al. Isolation of extracellular vesicles from stem cells. Methods Mol Biol 2017; 1660: 389-94.

27 Hu G, Yao H, Chaudhuri AD, Duan M, Yelamanchili SV, Wen H, et al. Exosome-mediated shuttling of microRNA-29 regulates HIV Tat and morphine-mediated neuronal dysfunction. Cell Death Dis 2012; 3: e381.

28 Chen L, Phillips MI, Miao HL, Zeng R, Qin G, Kim IM, et al. Infrared fluorescent protein 1.4 genetic labeling tracks engrafted cardiac progenitor cells in mouse ischemic hearts. PLoS One 2014; 9: e107841.

29 Wang Y, Zhou M, Wang X, Qin G, Weintraub NL, Tang Y. Assessing in vitro stem-cell function and tracking engraftment of stem cells in ischaemic hearts by using novel iRFP gene labelling. J Cell Mol Med 2014; 18: 1889-94.

30 Chen L, Pan Y, Zhang L, Wang Y, Weintraub N, Tang Y. Two-step protocol for isolation and culture of cardiospheres. Methods Mol Biol 2013; 1036: 75-80.

31 Kuzmichev A, Nishioka K, Erdjument-Bromage $\mathrm{H}$, Tempst $\mathrm{P}$, Reinberg D. Histone methyltransferase activity associated with a human multiprotein complex containing the Enhancer of Zeste protein. Genes Dev 2002; 16: 2893-905.

32 Pasini D, Malatesta M, Jung HR, Walfridsson J, Willer A, Olsson L, et al. Characterization of an antagonistic switch between histone $\mathrm{H} 3$ lysine 27 methylation and acetylation in the transcriptional regulation of Polycomb group target genes. Nucleic Acids Res 2010; 38: 495869.

33 He A, Ma Q, Cao J, von Gise A, Zhou P, Xie H, et al. Polycomb repressive complex 2 regulates normal development of the mouse heart. Circ Res 2012; 110: 406-15.

$34 \mathrm{Wu}$ J, Crowe DL. The histone methyltransferase EZH2 promotes mammary stem and luminal progenitor cell expansion, metastasis and inhibits estrogen receptor-positive cellular differentiation in a model of basal breast cancer. Oncol Rep 2015; 34: 455-60.

35 Tang Y, Wang Y, Chen L, Pan Y, Weintraub N. Cross talk between the Notch signaling and noncoding RNA on the fate of stem cells. Prog Mol Biol TransI Sci 2012; 111: 175-93.

36 Che CT, Wang ZJ, Chow MS, Lam CW. Herb-herb combination for therapeutic enhancement and advancement: theory, practice and future perspectives. Molecules 2013; 18: 5125-41.

37 Rider MA, Hurwitz SN, Meckes DG Jr. ExtraPEG: A polyethylene glycolbased method for enrichment of extracellular vesicles. Sci Rep 2016; 6: 23978 . 